Check for updates

Cite this: RSC Adv., 2017, 7, 21328

Received 11th February 2017

Accepted 9th April 2017

DOI: $10.1039 / \mathrm{c} 7 \mathrm{ra01742a}$

rsc.li/rsc-advances

\section{Iminoboronate-based dual-responsive micelles via subcomponent self-assembly for hydrophilic 1,2- diol-containing drug delivery $\dagger$}

\author{
Rujiang Ma, (D) ab Chuan Zhang, ${ }^{a}$ Yong Liu, ${ }^{a}$ Chang Li, ${ }^{a}$ Yanling $\mathrm{Xu}{ }^{\mathrm{c}}$ Baoxin Li, ${ }^{\mathrm{d}}$ \\ Yunliang Zhang, ${ }^{\star d}$ Yingli $\mathrm{An}^{\mathrm{a}}$ and Linqi Shi iD *ab
}

\begin{abstract}
Amphiphilic block copolymer micelles in aqueous solution have been used extensively in delivery of hydrophobic drugs with the hydrophobic core serving as the reservoir. However, encapsulation of hydrophilic drugs by polymeric micelles with hydrophobic core is relatively difficult because of the poor interaction between them. Herein, we report a novel kind of $\mathrm{pH} / \mathrm{GSH}$ dual-responsive complex micelles with a hydrophilic drug loaded, via direct subcomponent self-assembly of block copolymer poly(ethyleneglycol)-block-poly(L-lysine) (PEG-b-PLys), 2-formylbenzeneboronic acid (2-FPBA) and hydrophilic 1,2-diol-containing drugs (e.g. capecitabine (CAPE)) under physiological pH 7.4 based on the synergistic formation of an iminoboronate structure. The PEG-b-PLys/2-FPBA micelles without CAPE are well-characterized in terms of micellization mechanism, size, morphology, $\mathrm{pH}-$ and GSHresponsiveness. Encapsulation of CAPE by forming PEG- $b$-PLys/2-FPBA/CAPE complex micelles via synergistic formation of the iminoboronate structure is discussed and $\mathrm{pH}$ - and $\mathrm{GSH}$-responsive drugrelease studies are carried out. The complex micelles are stable under physiological neutral conditions but could be destroyed in response to the stimuli of physiological acidic condition (pH 5.0) and/or glutathione (GSH) at $\mathrm{pH}$ 7.4. $\mathrm{pH}$ - and GSH-responsive drug-releases are successfully achieved. The drug-loaded complex micelles could be endocytosed by HepG2 cells and efficiently deliver hydrophilic drugs into them. This type of complex micelles could be used as a promising platform for the delivery of hydrophilic 1,2-diol-containing drugs.
\end{abstract}

\section{Introduction}

Amphiphilic block copolymers, which are characterized by a hydrophilic block that is chemically tethered to a hydrophobic block, have been used extensively in pharmaceutical applications ranging from sustained-release technologies to gene delivery. ${ }^{1,2}$ The copolymers can self-assemble into polymeric micelles with a core/shell structure in aqueous solution. ${ }^{3,4}$ Upon micellization, the hydrophobic core regions serve as reservoirs for a variety of hydrophobic drugs, mainly

\footnotetext{
${ }^{a}$ State Key Laboratory of Medicinal Chemical Biology, Key Laboratory of Functional Polymer Materials of Ministry of Education, Institute of Polymer Chemistry, Nankai University, Tianjin 300071, China. E-mail: shilinqi@nankai.edu.cn

${ }^{b}$ Collaborative Innovation Center of Chemical Science and Engineering (Tianjin), Nankai University, Tianjin 300071, China

${ }^{c}$ Department of Biological Pharmacy, College of Basic Science, Tianjin Agricultural University, Tianjin 300384, China

${ }^{d}$ Endocrinology Department, Baoding First Central Hospital, Baoding, Hebei 071000, China. E-mail: bdzyl1228@163.com

$\dagger$ Electronic supplementary information (ESI) available: Synthesis and characterization of block copolymer, determination of the standard curve of CAPE, characterization of 2-FPBA binding to PEG- $b$-PLys by ${ }^{1} \mathrm{H}$ NMR spectra and loading of CAPE into micelles by UV-vis spectra. See DOI: 10.1039/c7ra01742a
}

depending on the hydrophobic interaction between the coreforming block and drugs. ${ }^{5-8}$ In addition, double hydrophilic block copolymers (DHBCs) were also developed to selfassemble into micelles for drug delivery based on the stimuliresponsive change of solubility of one block. Oren A. Scherman and co-workers synthesized a series of supramolecular DHBCs held together by cucurbit[8]uril (CB[8]) ternary complexation and the complex DHBCs were used to selfassemble into micelles at physiological temperature for encapsulation and stimuli-release of insulin, basic fibroblast growth factor (BFGF), and doxorubicin (DOX). ${ }^{9-11}$ However, amphiphilic block copolymer micelles do not work well in the encapsulation of hydrophilic drugs because of the weak interaction between the hydrophobic block and the hydrophilic drugs. Electrostatic reaction and covalent bonds are generally used to incorporate hydrophilic drugs into polymeric micelles by forming polyion complex (PIC) and prodrugs respectively., Kataoka and co-workers developed well-known PIC micelles for encapsulation of peptides and proteins via electrostatic interaction with PEGylated block copolymer poly(ethylene glycol)block-poly( $\alpha, \beta$-aspartic acid) (PEG- $b$-PAsp). ${ }^{\mathbf{1 3 , 1 4}}$ But the PIC system is vulnerable to salt concentration (ionic strength) and is only suitable for charge-rich drugs. As far as neutral 
hydrophilic drugs are concerned, a feasible way to incorporate them in polymeric micelles is conjugating to polymers via stimuli-responsive covalent bonds considering the nanocarrier stability during circulation and fast drug-release at target tissue. $^{\mathbf{1 2 , 1 5 - 1 7}}$ Recently, Herrera-Alonso et al. prepared a hydrophilic drug conjugated polymer complex based on boronic esterification between a diol-containing anticancer drug, capecitabine (CAPE), and phenylboronic acid-functionalized polycarbonates under anhydrous conditions. ${ }^{12} \mathrm{pH}$-sensitive dissociation of the cyclic boronate enabled release of CAPE from the self-assembled micelles. However, the preparation of polymer-drug conjugates generally involves relatively complicated synthetic chemistry, which may limit the application of block copolymer micelles in delivery of hydrophilic drugs. In fact, many FDA-approved 1,2- or 1,3-diol containing hydrophilic drugs such as capecitabine, dibromodulcitol, gemcitabine, ranimustine, and epigallocatechin gallate (EGCG) have been applied in anti-cancer therapy. It is desired to develop simple but effective methods to load and delivery this kind of neutral hydrophilic drugs by polymeric micelles.

Subcomponent self-assembly, introduced by Nitschke and co-workers, is a process which allows complex structures to be generated from simple building blocks. ${ }^{18-20}$ Usually, the complex structures are created from simple amine and aldehyde subcomponents by copper(I)-templated imine bond formation. ${ }^{19,20}$ Bunzen et al. prepared a kind of tunable multiresponsive gel with defined properties via self-assembly of subcomponents including 2-pyridinecarboxaldehyde, steroidal amine, and metal ions in one step. ${ }^{18}$ Nitschke et al. extended this "subcomponent self-assembly" concept to create new architectures with equimolar amounts of diol, amine, and 2formylphenylboronic acid (2-FPBA) based on the formation of an iminoboronate structure composed of imine bond and cyclic boronate. ${ }^{21}$ Subcomponent self-assembly not only allows the construction of complex structures in a very simple and direct way but also endows the resultant materials with multiresponsiveness because of the reversibility of the cyclic boronate and imine bond in response to stimuli.

However, polymeric micelles via subcomponent self-assembly have been rarely reported. In this study, we prepared a novel kind of dual-responsive micelles hydrophilic drug loaded via simple and direct subcomponent self-assembly of block copolymer poly(ethyleneglycol)-block-poly(L-lysine) (PEG-b-PLys), 2-FPBA and a hydrophilic 1,2-diol-containing drug, capecitabine (CAPE), under physiological pH 7.4 based on the synergistic formation of iminoboronate structure (Scheme 1). The micelles were stable under physiological neutral $\mathrm{pH}$, but could be destroyed in response to the stimulus of physiological acid ( $\mathrm{pH}$ 5.0) because of the breakage of the iminoboronate structure. ${ }^{1222-24}$ Additionally, the complex would undergo dissociation in the presence of glutathione (GSH), presumably because of the hydrolysis of the iminoboronate due to the disruption of the $\mathrm{B}-\mathrm{N}$ bond in response to $\mathrm{GSH}^{23-28}$ Based on these properties, $\mathrm{pH}$ and $\mathrm{GSH}$ responsive releases of CAPE were studied. The $\mathrm{pH}$ and $\mathrm{GSH}$ sensitive dissociation of the micelles can also be applied in a general manner to other 1,2-diol containing drugs, presenting a promising utility in biomedical field.

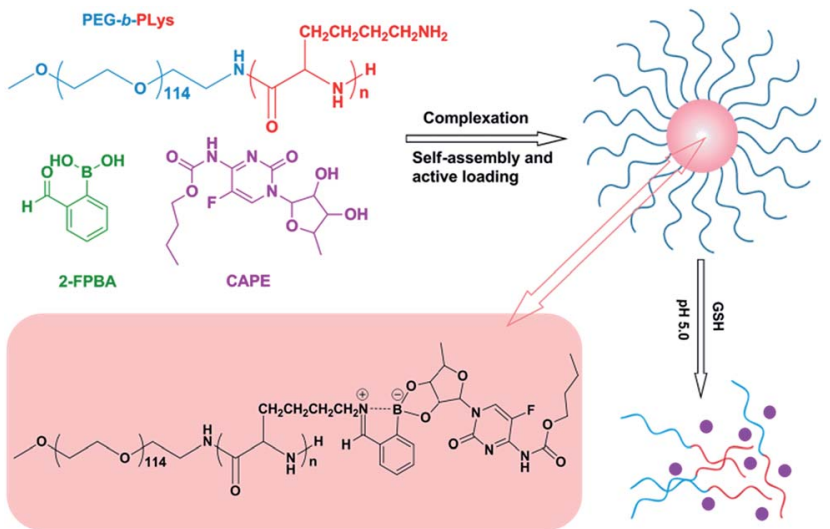

Scheme 1 Preparation of micelles with hydrophilic drug loaded by subcomponent self-assembly of $\mathrm{PEG}_{114}-b-\mathrm{PLys}_{44}, 2$-FPBA and CAPE in physiological $\mathrm{pH}$ and the $\mathrm{pH} / \mathrm{GSH}$-responsive properties of the selfassembled micelles.

\section{Experimental sections}

\subsection{Materials}

$\alpha$-Methoxy- $\omega$-aminopoly(ethylene glycol) $\left(\mathrm{CH}_{3} \mathrm{O}-\mathrm{PEG}_{114}-\mathrm{NH}_{2}\right.$; $\left.M_{\mathrm{w}}=5000 ; M_{\mathrm{w}} / M_{\mathrm{n}}=1.05\right)$ was purchased from Aladdin and used after dried under vacuum. 2-Formylbenzeneboronic acid (2-FPBA), capecitabine (CAPE), and fluorescein isothiocyanate (FITC) were purchased from Heowns without further purification. 1-Butylamine (1-BA) was purchased from General-Reagent. Block copolymer $\mathrm{PEG}_{114}-b$ - $\mathrm{PLys}_{44}$ was synthesized by polymerization of Lys(Z)-NCA initiated by the terminal amino group of PEG-NH ${ }_{2}$ (see ESI $\dagger$ for details about synthesis and characterization of the block copolymer). The FITC-labelled PEG- $b$-PLys block polymer was prepared by the reaction between the amino group of polymer and FITC in DMSO/ $\mathrm{H}_{2} \mathrm{O}$ solution. ${ }^{29}$ All of the buffer solutions used in this study were adjusted to an ionic strength of $150 \mathrm{mM}$ by addition of appropriate amount of $\mathrm{NaCl}$.

\subsection{Micellization of PEG-b-PLys with 2-FPBA}

$5 \mathrm{mg}$ of PEG- $b$-PLys was dissolved in $9 \mathrm{~mL}$ PBS buffer $(10 \mathrm{mM}$, $\mathrm{pH}$ 7.4) and stirred for $2 \mathrm{~h}$ before use. An equivalent 2-FPBA (3.10 mg) was dissolved in $1 \mathrm{~mL}$ PBS buffer (10 mM, pH 7.4) and then added to the polymer solution. The mixture was stirred at room temperature for $48 \mathrm{~h}$ for the formation of PEG- $b$-PLys/2FPBA micelles.

\subsection{Preparation of CAPE-loaded micelles}

The PEG- $b$-PLys/2-FPBA/CAPE complex micelles were prepared by self-assembly of the three subcomponents in aqueous solution of PBS 7.4. Firstly, $5 \mathrm{mg}$ of PEG- $b$-PLys was dissolved in 16 mL PBS buffer (10 mM, pH 7.4) and stirred for 2 h. Secondly, 2FPBA (3.10 mg, 1 equimolar to Lys units) and CAPE (7.42 mg, 1 equimolar to Lys units) were dissolved in the PBS 7.4 buffer respectively and these two solutions were added into the polymer solution. Then, the mixed solution was stirred at room temperature for $48 \mathrm{~h}$ for the formation of PEG- $b$-PLys/2-FPBA/ CAPE micelles. In order to remove unreacted 2-FPBA and 
CAPE, the micelle solution was processed by centrifugal ultrafiltration three times at the speed of $4000 \mathrm{rpm}$. Finally, the volume of the micelle solution was adjusted to $20 \mathrm{~mL}$ and the concentration of the micelles was $0.5 \mathrm{mg} \mathrm{mL}^{-1}$.

\subsection{Model reaction of 1-BA, 2-FPBA, and CAPE in $\mathrm{D}_{2} \mathrm{O}$ or DMSO}

To test the formation of iminoboronate between PEG- $b$-PLys, 2FPBA and CAPE, we evaluated the reaction between the model 1BA, 2-FPBA and CAPE in $\mathrm{D}_{2} \mathrm{O}$ and DMSO. Simply, $1 \mathrm{mg}$ 1-BA was dissolved in $500 \mu \mathrm{L} \mathrm{D}_{2} \mathrm{O}$ or DMSO followed by addition of $205 \mu \mathrm{L}$ 2-FPBA ( $1 \mathrm{mg}$ per $100 \mu \mathrm{L}, \mathrm{D}_{2} \mathrm{O}$ or DMSO) and $490 \mu \mathrm{L}$ CAPE ( $1 \mathrm{mg}$ per $100 \mu \mathrm{L}, \mathrm{D}_{2} \mathrm{O}$ or DMSO) with a molar ratio of $n_{1-\mathrm{BA}}: n_{2-\mathrm{FPBA}^{-}}$ $: n_{\mathrm{CAPE}}=1: 1: 1$. The mixed solution was stirred at room temperature for $48 \mathrm{~h}$ to assure a complete reaction and was finally made to a concentration of $0.5 \mathrm{mg} \mathrm{mL} \mathrm{m}^{-1}$.

\subsection{CAPE release study}

The release of CAPE from the complex micelles was evaluated by incubating the drug-loaded micelles in $10 \mathrm{mM}$ buffer with different $\mathrm{pH}$ values and various GSH concentrations at $37{ }^{\circ} \mathrm{C}$. Typically, $2 \mathrm{~mL}$ CAPE-loaded micelle solution was injected in a dialysis bag with a molecular weight cutoff of $3.5 \mathrm{kDa}$, then the dialysis bag was immersed in $20 \mathrm{~mL}$ PBS 7.4 buffer with varying GSH concentrations of 0,10 and $20 \mathrm{mM}$ or $20 \mathrm{~mL}$ citric acid buffer solution at $\mathrm{pH} 5.0$ respectively. At certain time intervals, 1 $\mathrm{mL}$ of the buffer solution was taken to measure the CAPE concentration and $1 \mathrm{~mL}$ fresh buffer was added. The standard curve for the calibration of CAPE concentration was measured on a UV-vis spectrophotometer and details were included in the ESI (Fig. S2†).

\subsection{Cellular uptake study}

The cellular uptake behavior of PEG- $b$-P(Lys-co-LysFITC)/2FPBA/CAPE complex micelles were investigated by confocal laser scanning microscopy (CLSM) using HepG2 cells. The cells were plated on microscope slides in a 24 -well plate $(1 \times 105$ cells per well) under $5 \% \mathrm{CO}_{2}$ atmosphere at $37^{\circ} \mathrm{C}$, using DMEM medium supplemented with $10 \%$ fetal bovine serum, antibiotics penicillin (100 IU mL $\left.\mathrm{mL}^{-1}\right)$, and streptomycin $\left(100 \mu \mathrm{g} \mathrm{mL} \mathrm{m}^{-1}\right)$ for $24 \mathrm{~h}$. Then the cells were exposed to FITC labelled, CAPEloaded micelles for $4 \mathrm{~h}$ at $37{ }^{\circ} \mathrm{C}$ in a humidified $5 \% \mathrm{CO}_{2}$-containing atmosphere (FITC-micelles dosage: $50 \mu \mathrm{g} \mathrm{mL}{ }^{-1}$ ). After incubation, the culture medium was removed. The cells on microscope plates were fixed with $4 \%$ formaldehyde for $20 \mathrm{~min}$ and washed three times with PBS buffer. Then the cell nuclei were stained with 4,6-diamidino-2-phenylindole (DAPI) for 10 min and washed six times with PBS buffer. The fluorescence images were obtained using a confocal laser scanning microscope (TCS SP5).

\subsection{Characterizations}

The UV-vis spectra were recorded on a UV-2550 ultravioletvisible spectrophotometer (Shimadzu, Japan). ${ }^{1} \mathrm{H}$ NMR and

${ }^{11} \mathrm{~B}$ NMR spectra were measured on a Varian UNITY-plus $400 \mathrm{M}$
NMR spectrometer at room temperature. Dynamic light scattering (DLS) experiments including the determination of size distributions and the monitoring of light scattering intensities (LSI) at the scattering angle of $90{ }^{\circ} \mathrm{C}$ were performed on a laser light scattering spectrometer (BI-200SM) equipped with a digital correlator (BI-9000AT) at $636 \mathrm{~nm}$ at $37^{\circ} \mathrm{C}$. About $1 \mathrm{~mL}$ of micelle solution was filtered into a clean scintillation vial through a 0.8 $\mu \mathrm{m}$ Millipore filter for DLS measurements. Transmission electron microscopy (TEM) measurements were performed using a Philips T20ST electron microscope at an acceleration voltage of $100 \mathrm{kV}$. To prepare the TEM samples, the micelle solution was first diluted and then dropped onto a carbon-coated copper grid and dried slowly at room temperature. The drug-loaded micelles were processed by TG16-WS high speed centrifuge to remove the unreacted small molecules.

\section{Results and discussion}

\subsection{Formation of PEG-b-PLys/2-FPBA and PEG- $b$-PLys/2- FPBA/CAPE micelles}

The reaction between PEG- $b$-PLys and 2-FPBA in PBS buffer (10 mM, pH 7.4) was monitored by UV-vis. As shown in Fig. 1A, when 2-FPBA solution was added into the polymer solution, the intensity of the absorption strength at $286 \mathrm{~nm}$ increased as time
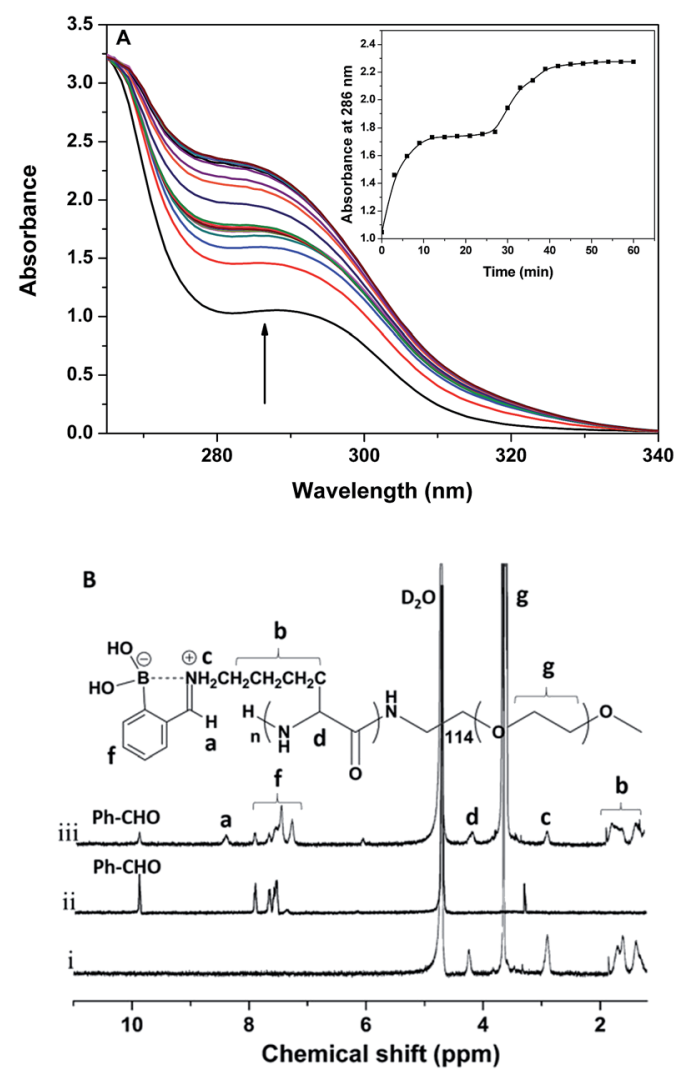

Fig. 1 (A) UV-vis absorption spectra of the solution mixture of PEG- $b$ PLys and 2-FPBA in PBS buffer ( $\mathrm{pH} 7.4)$ as a function of time with an interval of $3 \mathrm{~min}$, inset shows the absorption at $286 \mathrm{~nm}$ as a function of time. (B) The ${ }^{1} \mathrm{H}$ NMR spectra of PEG- $b$-PLys (i), 2-FPBA (ii) and the mixture of PEG- $b$-PLys and 2-FPBA (iii) in $\mathrm{D}_{2} \mathrm{O}$. 
went by. The observed spectral change can be attributed to the imine formation by the aldehyde group on 2-FPBA and the $\omega$ amino group on Lys unit. ${ }^{30}$ The inset of the absorbance at $286 \mathrm{~nm}$ as a function of reaction time in Fig. 1A demonstrated the kinetics of the reaction and it completed within $60 \mathrm{~min}$ as indicated by the invariable intensity of the absorption band finally. Further evidence supporting the imine formation came from ${ }^{1} \mathrm{H}$ NMR study. As shown in Fig. 1B, the signal of aldehyde protons on 2-FPBA at 9.9 ppm decreased significantly after reaction with PEG- $b$-PLys and the ratio of integrated areas from the aldehyde protons at $9.9 \mathrm{ppm}$ to those from the aromatic protons in the range of 7.2-8.0 ppm reduced from $0.98 / 4.00$ to $0.39 / 4.00$, indicating that about $60 \%$ 2-FPBA were bound to PEG- $b$-PLys (see ESI Fig. S3 $\dagger$ ). A new signal appeared at $8.4 \mathrm{ppm}$ could be attributed to the protons on benzoic imine conjugate structure. $^{22,30}$ At the same time, the signals of protons on the side alkyl chain of the PLys block merged into two broad signals near 1.2-2.0 ppm. It was a typical feature of protons on polymers with restricted mobility ${ }^{\mathbf{1 5 , 3 3}}$ because of the resultant PLys/ 2-FPBA complex was relatively hydrophobic at $\mathrm{pH}$ 7.4.

The PEG- $b$-PLys/2-FPBA complex was observed to selfassemble into polymeric micelles under the $\mathrm{pH}$ ranging from 6.5-7.4 and their size and morphology were characterized by DLS and TEM. The PEG- $b$-PLys/2-FPBA complex micelles had an average hydrodynamic size of $127 \mathrm{~nm}$ and a very narrow size distribution (Fig. 2A) with a polydispersity value of 0.085 . While the TEM image shown in Fig. 2B indicated irregular morphologies and a larger polydispersity than the DLS result for the PEG$b$-PLys/2-FPBA micelles, which may be attributed to unavoidable aggregation or fusion of micelles on the carbon film upon drying
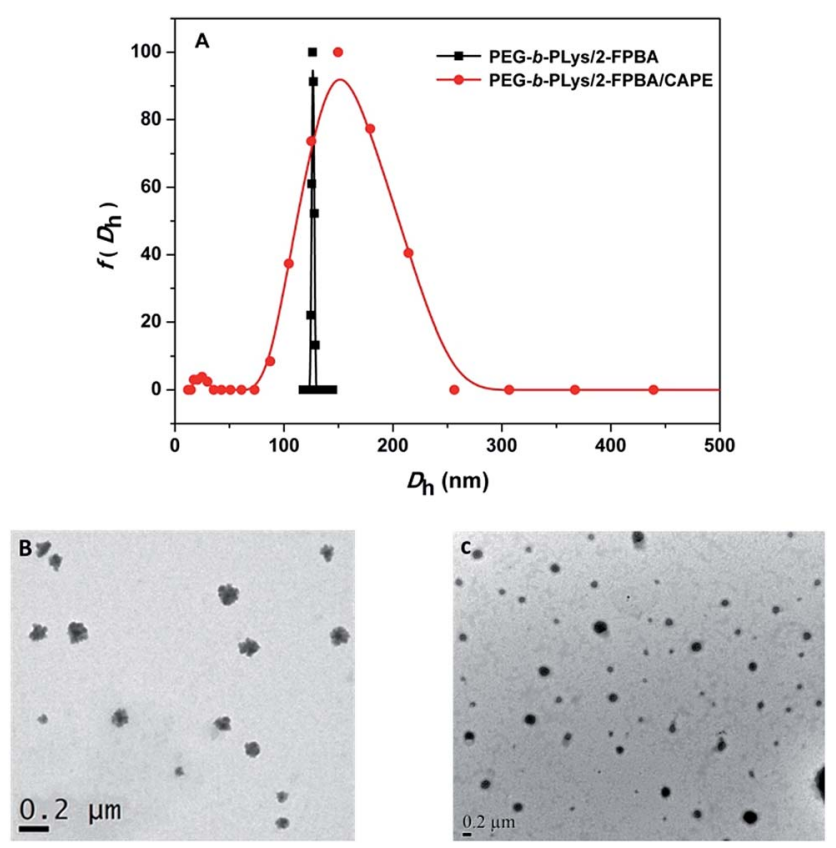

Fig. 2 (A) Size distributions of PEG- $b$-PLys/2-FPBA and PEG-b-PLys/ 2-FPBA/CAPE complex micelles. (B) TEM image of PEG- $b$-PLys/2FPBA complex micelles. (C) TEM image of PEG- $b$-PLys/2-FPBA/CAPE complex micelles. the TEM sample. When the 1,2-diol-containing drug, CAPE, was simultaneously mixed with PEG- $b$-PLys and 2-FPBA, the three components similarly self-assembled into drug-loaded complex micelles, which had a relatively broad size distribution (polydispersity value $=0.223$ ) with an average hydrodynamic size of $152 \mathrm{~nm}$ (Fig. 2A). TEM image (Fig. 2C) indicated spherical morphologies for the three-component complex micelles. It was reported that 2-FPBA had an apparent $\mathrm{p} K_{\mathrm{a}}$ value around 7.3, which may endow itself with a strong inclination to conjugate the 1,2-diol on CAPE and result in stable cyclic boronate under physiological $\mathrm{pH}$ 7.4. In fact, the combination between the aldehyde group on 2-FPBA and the $\omega$-amino group on PLys block via imine bond may further facilitate the formation of boronate between 2-FPBA and CAPE, synergistically leading to an iminoboronate structure. ${ }^{31,32}$ As a result, CAPE was anchored on the PLys block bridged by 2-FPBA. The resultant PEG- $b$-PLys/2-FPBA/ CAPE complex behaved like amphiphilic block copolymer with a hydrophilic PEG block and a relatively hydrophobic PLys/2FPBA/CAPE block and could self-assemble into core-shell micelles. Thus the hydrophilic CAPE was easily and successfully encapsulated into the complex micelles.

To identify the encapsulation of CAPE by the synergistic formation of iminoboronate structure among PEG- $b$-PLys, 2FPBA and CAPE, UV-vis spectra were recorded for free CAPE, polymer complex PEG- $b$-PLys/2-FPBA, and drug-loaded polymer complex PEG- $b$-PLys/2-FPBA/CAPE. The appearance of the characteristic signal of anchored CAPE at $304 \mathrm{~nm}$ was observed for PEG- $b$-PLys/2-FPBA/CAPE complex by UV-vis spectrometry (Fig. S4, ESI $\dagger$ ). For more details, a model reaction was used with PEG- $b$-PLys replaced by 1-butylamine (1-BA) as illustrated in Scheme 2 and examined by ${ }^{1} \mathrm{H}$ NMR and ${ }^{11} \mathrm{~B}$ NMR technologies in deuteroxide water $\left(\mathrm{D}_{2} \mathrm{O}\right)$ and dimethyl sulfoxide (DMSO) respectively. As shown in Fig. 3A for 2-FPBA, reaction with 1-BA and CAPE led to the complete disappearance of the signal of aldehyde protons at $9.9 \mathrm{ppm}$, indicating that all of the aldehyde groups were converted. A new signal corresponding to the protons on the benzoic imine conjugate structure appeared at $8.5 \mathrm{ppm},{ }^{22,30}$ indicating the combination between 1-BA and 2FPBA. While in the ${ }^{11} \mathrm{~B}$ NMR spectra in Fig. 3B, 2-FPBA itself showed two signals at $31.6 \mathrm{ppm}$ and $-3.6 \mathrm{ppm}$, indicating that it existed in two forms of a neutral $\mathrm{R}-\mathrm{B}(\mathrm{OH})_{2}$ and an anionic R$\mathrm{B}(\mathrm{OH})_{3}{ }^{-}$respectively. ${ }^{30,33}$ When 2-FPBA was mixed with 1-BA, the signal at $31.6 \mathrm{ppm}$ almost completely disappeared and a new peak at $8.9 \mathrm{ppm}$ appeared, which could be assigned to the resultant $\mathrm{N}-\mathrm{B}$ coordinated tetrahedral boron. ${ }^{34}$ This demonstrated that the introduction of nitrogen atom adjacent the boronic acid via formation of imine bond by aldehyde and amino group induced most of the neutral $\mathrm{R}-\mathrm{B}(\mathrm{OH})_{2}$ being transformed into the anionic $\mathrm{R}-\mathrm{B}(\mathrm{OH})_{3}{ }^{-}$which had a stronger tendency to combine 1,2-diols. ${ }^{23}$ When CAPE was further added into the 1-BA/2-FPBA system, the peak of the $\mathrm{N}-\mathrm{B}$ coordinated tetrahedral boron moved from $8.9 \mathrm{ppm}$ to $14.4 \mathrm{ppm}$, indicating the formation of cyclic boronate. ${ }^{34}$ The $\mathrm{N}-\mathrm{B}$ coordination was favourable for the formation of boronate, and in return the boronate also could promote the $\mathrm{N}-\mathrm{B}$ coordination by raising the electrophilicity, or the Lewis acidity, of the boron atom. ${ }^{34,35}$ Thus, the iminoboronate structure was synergistically obtained. 


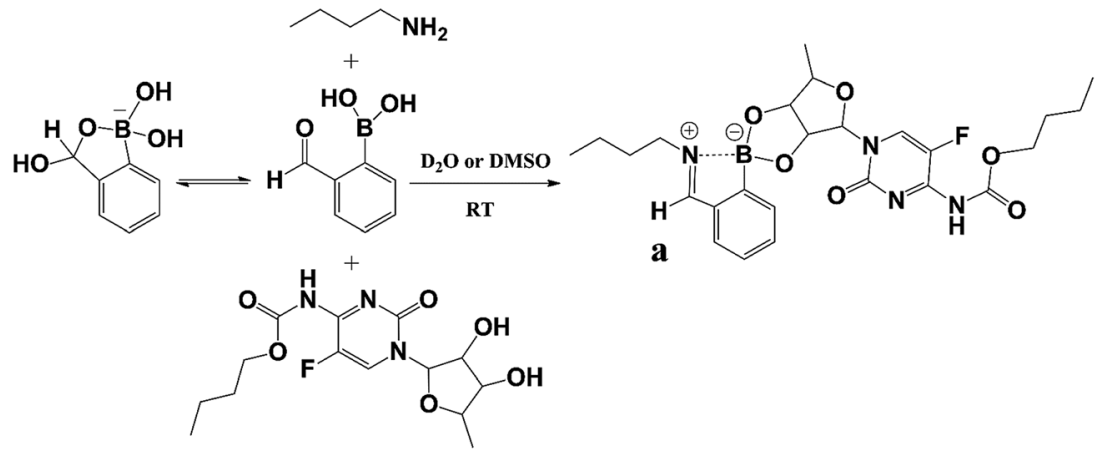

Scheme 2 Model reaction of 1-BA with 2-FPBA and CAPE in $\mathrm{D}_{2} \mathrm{O}$ or DMSO.

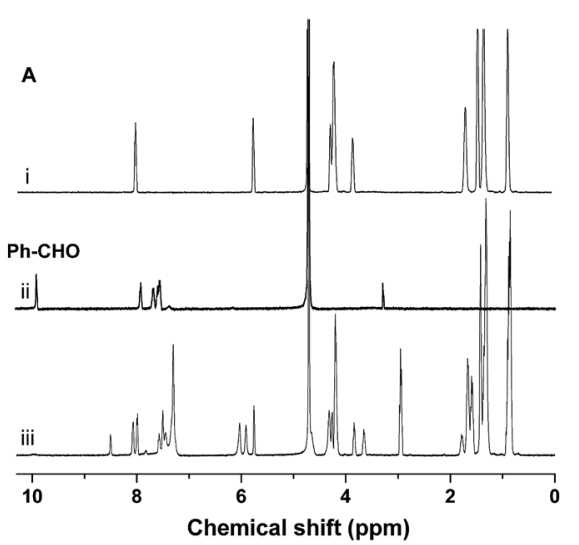

B
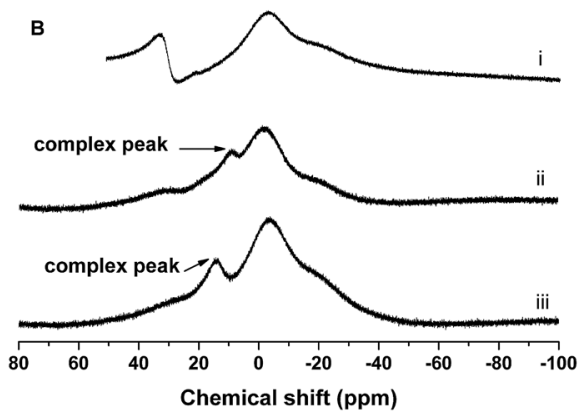

Fig. 3 (A) ${ }^{1} \mathrm{H}$ NMR spectra of CAPE (i), 2-FPBA (ii) and 1-BA/2-FPBA/ CAPE complex (iii) in $\mathrm{D}_{2} \mathrm{O}$; (B) ${ }^{11} \mathrm{~B}$ NMR spectra of 2-FPBA (i), 1-BA/2FPBA (ii) and 1-BA/2-FPBA/CAPE complex (iii) in deuterated DMSO.

\section{2. pH-responsiveness of PEG-b-PLys/2-FPBA micelles}

Imine bond formed by aldehyde and primary amine was a wellknown $\mathrm{pH}$-sensitive dynamic covalent bond that was stable at neutral pH 7.4 but could be destroyed under weak acid conditions such as the endosomal $\mathrm{pH} 5.0 .^{22}$ This laid the foundation for $\mathrm{pH}$-responsive intracellular delivery of polymer-conjugated anticancer drugs by polymeric nanocarriers. ${ }^{1}$ In this study, pH-responsiveness of the PEG- $b$-PLys/2-FPBA micelles was investigated by monitoring the variations of relative light scattering intensity (LSI) and micelle sizes in response to $\mathrm{pH}$ changes in aqueous solutions. As shown in Fig. 4A, the constant LSI of the micelle solution at $\mathrm{pH} 7.4$ indicated the stability of the
PEG- $b$-PLys/2-FPBA micelles under the physiological neutral condition. When the citric acid buffer at pH 5.0 was used, the LSI of the micelle solution decreased gradually to $40 \%$ of the initial intensity in $2 \mathrm{~h}$, suggesting the dissociation of the PEG- $b$ PLys/2-FPBA micelles, which may be caused by the hydrolysis of the imine bond under the weak acid condition. Fig. 4B showed the size variation of the PEG- $b$-PLys/2-FPBA micelles at pH 5.0 as a function of time. The decreasing average hydrodynamic
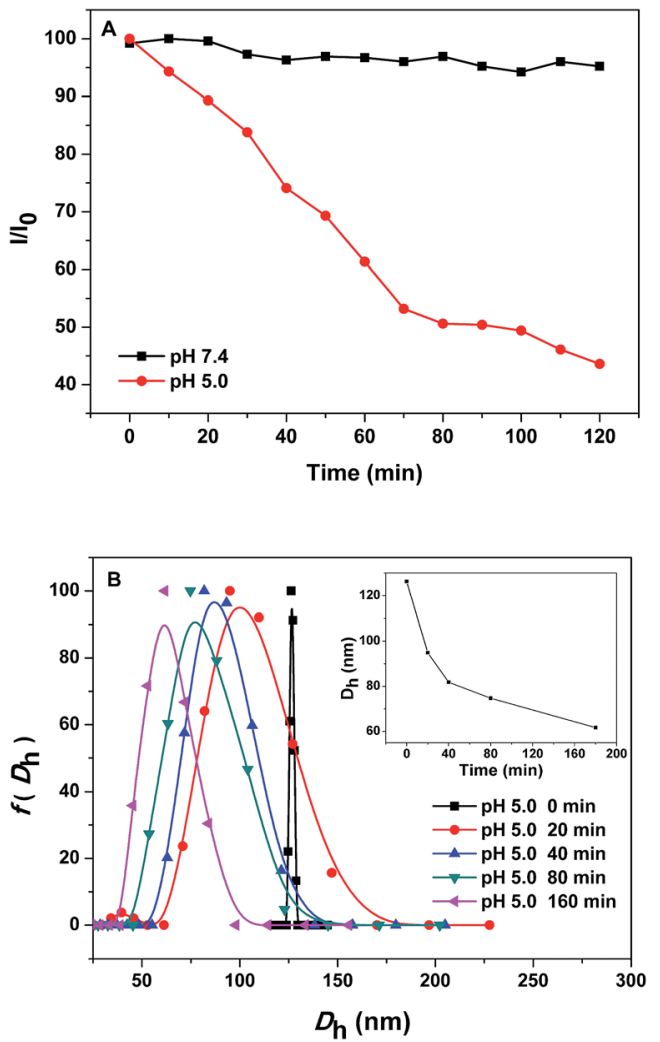

Fig. 4 (A) $\mathrm{pH}$-responsiveness of the PEG-b-PLys/2-FPBA micelles in aqueous solutions of PBS 7.4 and citric acid buffer ( $\mathrm{pH}$ 5.0) in terms of normalized light scattering intensity as a function of time. (B) Size variation of the PEG- $b$-PLys/2-FPBA micelles in aqueous solution of citric acid buffer ( $\mathrm{pH}$ 5.0) as a function of time, inset shows the change of the average particle size as a function of time (B). All of the solutions had a micelle concentration of $0.4 \mathrm{mg} \mathrm{mL}^{-1}$. 
diameter $\left(D_{\mathrm{h}}\right)$ from $126 \mathrm{~nm}$ at the initial time (the status of micelles at this moment could be considered the same as that at $\mathrm{pH} 7.4$ ) to $62 \mathrm{~nm}$ in $3 \mathrm{~h}$ (inset in Fig. 4B) also suggested the decomposition of the PEG- $b$-PLys/2-FPBA micelles.

\subsection{GSH-responsiveness of the PEG- $b$-PLys/2-FPBA micelles}

Since the PEG- $b$-PLys/2-FPBA micelles were prepared based on the anchoring of 2-FPBA on PEG- $b$-PLys via the reversible imine bond which was stabilized by the $\mathrm{N}-\mathrm{B}$ coordination, ${ }^{25}$ it could be possible that the micelles were disrupted by addition of glutathione (GSH) as it could destroy the N-B coordination and thus promote the imine hydrolysis. ${ }^{25,36}$ This offered the PEG- $b$ PLys/2-FPBA micelles with GSH-responsiveness. Fig. 5A showed the variations of LSI of the PEG- $b$-PLys/2-FPBA micelle solutions with different GSH concentrations $(5,10$, and $20 \mathrm{mM})$ as a function of time. The LSI decreased quickly at the initial stage and then levelled off, which was different from the gradual decrease as the case of pH 5.0 condition (Fig. 4A), indicating the PEG- $b$-PLys/2-FPBA micelles could fast response to GSH. Higher GSH concentration led to lower LSI, suggesting more micelles were disrupted. Fig. 5B showed the size distributions of the PEG- $b$-PLys/2-FPBA micelles in aqueous solution with $10 \mathrm{mM}$ GSH at $0 \mathrm{~min}$ (the status of micelles at this moment could be considered the same as that at $\mathrm{pH} 7.4$ without GSH) and
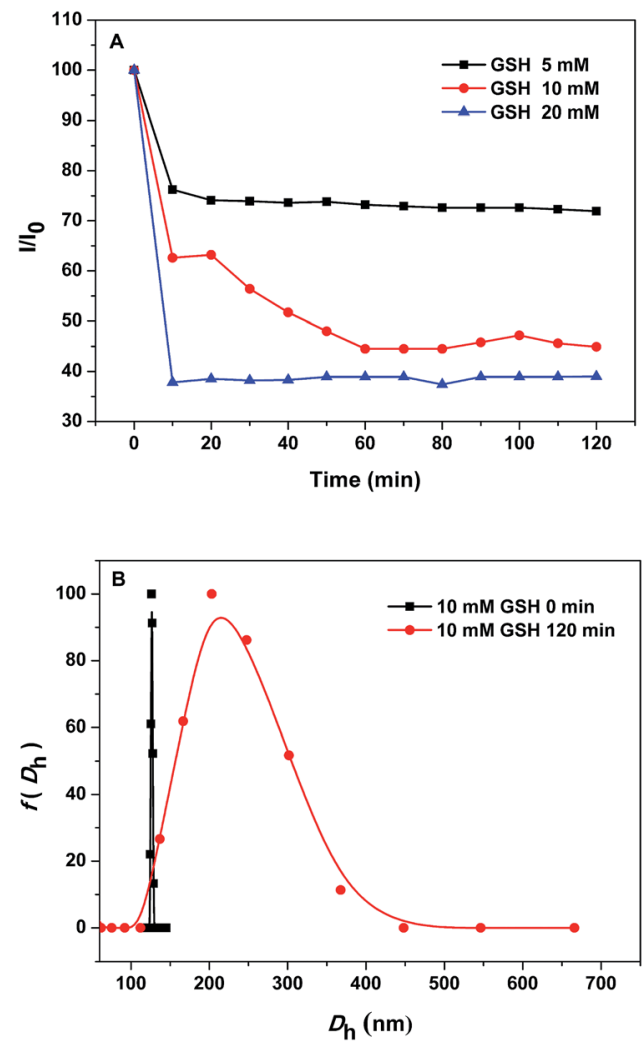

Fig. 5 (A) GSH-responsiveness of the PEG- $b$-PLys/2-FPBA micelles in aqueous solutions of PBS 7.4 in terms of normalized light scattering intensity as a function of time. (B) Size distributions of the PEG-b-PLys/ 2 -FPBA micelles in aqueous solution with $10 \mathrm{mM} \mathrm{GSH}$ at 0 and $120 \mathrm{~min}$
$120 \mathrm{~min}$. The increase of $D_{\mathrm{h}}$ from the initial $127 \mathrm{~nm}$ to the later $215 \mathrm{~nm}$ with a broader size distribution may be caused by the micelles experiencing a swelling process before dissociation.

\section{4. $\quad \mathrm{pH} / \mathrm{GSH}-$ responsive release of CAPE}

The $\mathrm{pH}$ - and GSH-responsiveness discussed above made the PEG- $b$-PLys/2-FPBA micelles as promising nanocarriers for intracellular delivery of anticancer drugs, ${ }^{37,38}$ especially those bearing 1,2-diols. Herein, capecitabine (CAPE) was used as a hydrophilic 1,2-diol-containing model drug to be encapsulated into the PEG- $b$-PLys/2-FPBA/CAPE micelles via subcomponent self-assembly and $\mathrm{pH}^{-}$and GSH-responsive drugreleases were carried out. As discussed above, the iminoboronate structure was synergistically formed with imine bond, N-B coordination, and cyclic boronate, and they were promoted each other. It was possible to hydrolyze the boronate by destroying either the imine bond or the $\mathrm{N}-\mathrm{B}$ coordination by weak acid or GSH respectively and thus to release CAPE upon the dissociation of the PEG- $b$-PLys/2-FPBA/CAPE micelles. Fig. 6 showed the $\mathrm{pH}$ - and GSH-responsive CAPE release profiles under different conditions. The cumulative drug-release from the complex micelles was calculated by monitoring the UV-vis absorption changes at $310 \mathrm{~nm}$ of CAPE in the external fluid. It was shown in Fig. 6A that the release rate was faster at $\mathrm{pH} 5.0$ than that at neutral $\mathrm{pH} 7.4$ with the cumulated CAPE release of $92.9 \%$ and $70.0 \%$ respectively in $24 \mathrm{~h}$, indicating a faster and
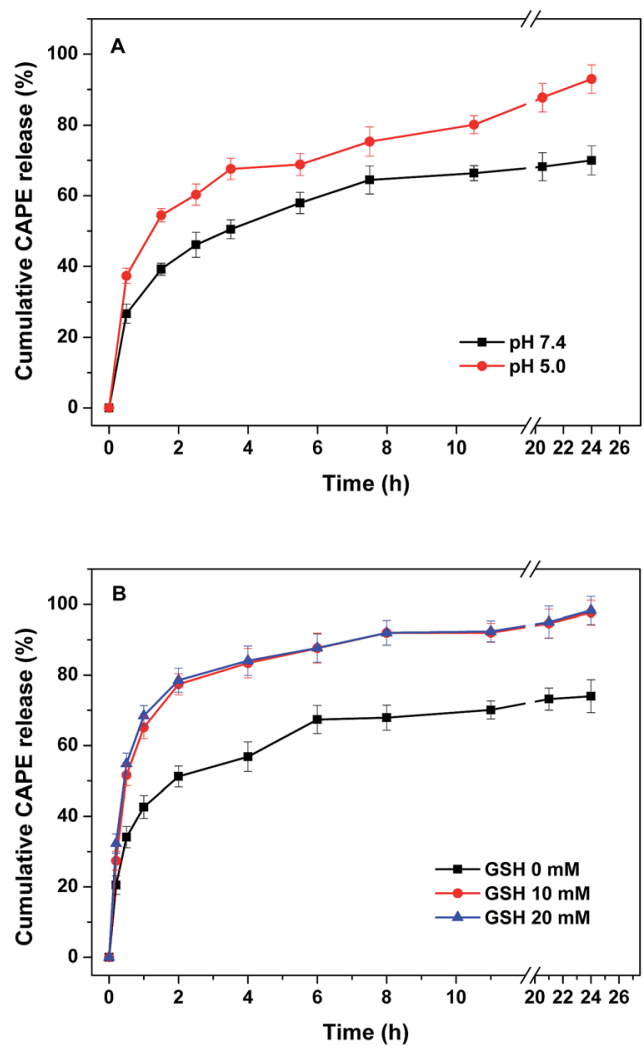

Fig. 6 (A) $\mathrm{pH}$-responsive release of CAPE from complex micelles in buffers at $\mathrm{pH} 5.0$ and 7.4. (B) GSH-responsive release of CAPE from complex micelles with varying concentrations of GSH at pH 7.4. 
nearly complete dissociation of the complex micelles in response to weak acid at pH 5.0. When GSH was used at neutral $\mathrm{pH}$ 7.4, faster drug-release was also observed than that without GSH with the cumulated CAPE release of $98.3 \%$ both for 10 and $20 \mathrm{mM}$ GSH in $24 \mathrm{~h}$, indicating complete dissociation of the drug-loaded complex micelles in response to GSH. The release profiles under 10 and $20 \mathrm{mM}$ GSH were exactly overlapped, suggesting that the physiological GSH concentration of $10 \mathrm{mM}$ (ref. 37 and 38) was enough for triggering complete CAPE release from the complex micelles. The $\mathrm{pH}$ - and $\mathrm{GSH}$-responsive drug-releases make the PEG- $b$-PLys/2-FPBA/CAPE micelles as promising nanocarriers for intracellular delivery. It was noted that, under different conditions, the drug-release rates were fast in the initial $2 \mathrm{~h}$ and then slowed down in $24 \mathrm{~h}$, which may be attributed to that both the imine bond and boronate were of dynamic covalent ones that were sensitive to continuous dilution by dialysis against the external fluid. This moved the equilibrium towards the direction of the hydrolysis of the iminoboronate and thus destroyed the drug-loaded micelles. We currently found that multi-catechol-containing molecules such as epigallocatechin gallate (EGCG) and tannin acid could also be co-encapsulated into the PEG- $b$-PLys/2-FPBA/CAPE micelles via more stronger interaction between catechol moiety and boronic acid..$^{39,40}$ This may not only increase micelle stability because of possible cross-linking effect and ameliorate drugrelease profile but also provide a feasible way to co-delivery of more than one hydrophilic drugs for enhancing anti-tumor effects as both EGCG and tannin acid are active in anticancer therapy. Further study is now being carried out focusing on EGCG or tannin acid co-encapsulated PEG- $b$-PLys/2-FPBA/CAPE system with increased micelle stability, ameliorated drugrelease profile, and enhanced anticancer effect.

\subsection{Cellular uptake of CAPE-loaded micelles}

The cellular uptake behavior of FITC labeled, CAPE loaded complex micelles in human HepG2 cells was investigated using CLSM (Fig. 7). Clearly, distinct FITC fluorescence was observed in HepG2 cells after incubation with FITC labelled complex micelles for $4 \mathrm{~h}$, which demonstrated that the CAPEloaded complex micelles can be endocytosed by cancer cells efficiently.
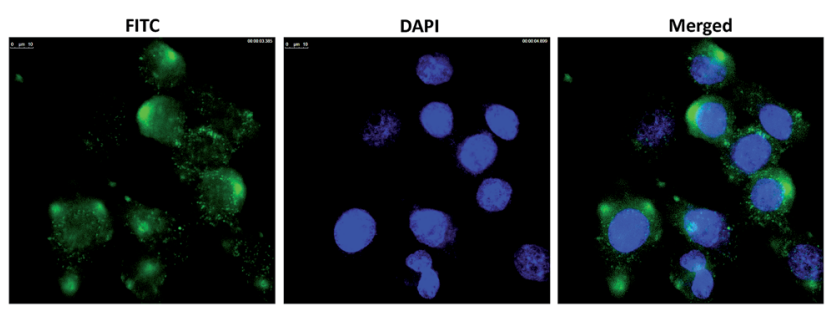

Fig. 7 CLSM images of HepG2 cells incubated with FITC-labeled, drug-loaded micelles for $4 \mathrm{~h}$. The images from left to right show FITC fluorescence in cells (green), cell nuclei stained by DAPI (blue), and overlays of the two images. The scale bars correspond to $10 \mu \mathrm{m}$ in all images.

\section{Conclusions}

In conclusion, we present herein a direct and simple way to prepare dual-responsive complex micelles for loading of hydrophilic drugs via a simple subcomponent self-assembly of block copolymer PEG- $b$-PLys, 2-FPBA and a 1,2-diol-containing drug CAPE under physiological $\mathrm{pH} 7.4$ based on the synergistic formation of iminoboronate. The complex micelles are stable under physiological neutral condition but could be destroyed in response to the stimuli of physiological acid condition $(\mathrm{pH}$ 5.0) and/or glutathione (GSH) because of the breakage of the iminoboronate structure. $\mathrm{pH}^{-}$and $\mathrm{GSH}-$ responsive drug-releases are successfully achieved. Also, the drug-loaded complex micelles can be endocytosed by HepG2 cells and efficiently deliver hydrophilic drugs into them. The features of easy and convenient encapsulation of 1,2-diol containing drugs via simple subcomponent self-assembly as well as physiological acid $\mathrm{pH}^{-}$and GSH-responsiveness make the complex micelles as promising nanocarriers for intracellular delivery of hydrophilic 1,2-diol-containing anticancer drugs.

\section{Acknowledgements}

This study was financially supported by the National Natural Science Foundation of China (No. 21274001, 51390483, 91527306, 21620102005, and 21404082), the Natural Science Foundation of Tianjin, China (No. 15JCYBJC29700) and the PCSIRT (IRT1257).

\section{References}

1 Z. H. Ge and S. Y. Liu, Chem. Soc. Rev., 2013, 42, 7289-7325.

2 R. J. Ma and L. Q. Shi, Functional Polymers for Nanomedicine, 2013, pp. 207-226.

3 K. Kataoka, A. Harada and Y. Nagasaki, Adv. Drug Delivery Rev., 2001, 47, 113-131.

4 M. L. Adams, A. Lavasanifar and G. S. Kwon, J. Pharm. Sci., 2003, 92, 1343-1355.

5 G. Gaucher, M. H. Dufresne, V. P. Sant, N. Kang, D. Maysinger and J. C. Leroux, J. Controlled Release, 2005, 109, 169-188.

6 A. Rösler, G. W. M. Vandermeulen and H. A. Klok, Adv. Drug Delivery Rev., 2012, 64, 270-279.

7 R. J. Ma, X. C. Sun, X. J. Liu, Y. L. An and L. Q. Shi, Aust. J. Chem., 2014, 67, 127-133.

8 T. J. Yin, J. Wang, L. F. Yin, L. J. Shen, J. P. Zhou and M. R. Huo, Polym. Chem., 2015, 6, 8047-8059.

9 X. J. Loh, M. H. Tsai, J. D. Barrio, E. A. Appel, T. C. Lee and O. A. Scherman, Polym. Chem., 2012, 3, 3180.

10 X. J. Loh, J. del Barrio, T. C. Lee and O. A. Scherman, Chem. Commun., 2014, 50, 3033-3035.

11 X. J. Loh, J. del Barrio, P. P. Toh, T. C. Lee, D. Jiao, U. Rauwald, E. A. Appel and O. A. Scherman, Biomacromolecules, 2012, 13, 84-91.

12 Y. E. Aguirre-Chagala, J. L. Santos, Y. X. Huang and M. Herrera-Alonso, ACS Macro Lett., 2014, 3, 1249-1253. 
13 A. Harada and K. Kataoka, J. Am. Chem. Soc., 1999, 121, 92419242.

14 A. Harada and K. Kataoka, J. Controlled Release, 2001, 72, 8591.

15 N. Larson and H. Ghandehari, Chem. Mater., 2012, 24, 840853.

16 S. A. Senevirathne, K. E. Washington, M. C. Biewer and M. C. Stefan, J. Mater. Chem., 2016, 4, 360-370.

17 R. Duncan, Nat. Rev. Drug Discovery, 2003, 2, 347-360.

18 H. Bunzen, Nonappa, E. Kalenius, S. Hietala and E. Kolehmainen, Chem.-Eur. J., 2013, 19, 12978-12981.

19 V. E. Campbell, R. Guillot, E. Riviere, P. T. Brun, W. Wernsdorfer and T. Mallah, Inorg. Chem., 2013, 52, 5194-5200.

20 J. R. Nitschke, Acc. Chem. Res., 2007, 40, 103-112.

21 M. Hutin, G. Bernardinelli and J. R. Nitschke, Chem.-Eur. J., 2008, 14, 4585-4593.

22 C. Wang, G. T. Wang, Z. Q. Wang and X. Zhang, Chem.-Eur. J., 2011, 17, 3322-3325.

23 R. J. Ma, H. Yang, Z. Li, G. Liu, X. C. Sun, X. J. Liu, Y. L. An and L. Q. Shi, Biomacromolecules, 2012, 13, 3409-3417.

24 G. A. Ellis, M. J. Palte and R. T. Raines, J. Am. Chem. Soc., 2012, 134, 3631-3634.

25 P. M. Cal, J. B. Vicente, E. Pires, A. V. Coelho, L. F. Veiros, C. Cordeiro and P. M. Gois, J. Am. Chem. Soc., 2012, 134, 10299-10305.

26 P. M. Cal, R. F. Frade, C. Cordeiro and P. M. Gois, Chem.-Eur. J., 2015, 21, 8182-8187.
27 C. T. Hoang, I. Prokes, G. J. Clarkson, M. J. Rowland, J. H. Tucker, M. Shipman and T. R. Walsh, Chem. Commun., 2013, 49, 2509-2511.

28 P. M. Cal, R. F. Frade, V. Chudasama, C. Cordeiro, S. Caddick and P. M. Gois, Chem. Commun., 2014, 50, 5261-5263.

29 H. J. Gao, J. Xiong, T. J. Cheng, J. J. Liu, L. P. Chu, J. F. Liu, R. J. Ma and L. Q. Shi, Biomacromolecules, 2013, 14, 460-467.

30 N. J. Gutierrez-Moreno, F. Medrano and A. K. Yatsimirsky, Org. Biomol. Chem., 2012, 10, 6960-6972.

31 M. Hutin, G. Bernardinelli and J. R. Nitschke, Chem.-Eur. J., 2008, 14, 4585-4593.

32 D. A. Tickell, M. F. Mahon, S. D. Bull and T. D. James, Org. Lett., 2013, 15, 860-863.

33 H. Otsuka, E. Uchimura, H. Koshino, T. Okano and K. Kataoka, J. Am. Chem. Soc., 2003, 125, 3493-3502.

34 L. Zhu, S. H. Shabbir, M. Gray, V. M. Lynch, S. Sorey and E. V. Anslyn, J. Am. Chem. Soc., 2006, 128, 1222-1232.

35 S. Hermanek, Chem. Rev., 1992, 92, 325-362.

36 P. M. Cal, R. F. Frade, C. Cordeiro and P. M. Gois, Chem.-Eur. J., 2015, 21, 1-7.

37 L. L. Wu, Y. Zou, C. Deng, R. Cheng, F. H. Meng and Z. Y. Zhong, Biomaterials, 2013, 34, 5262-5272.

38 Y. Kang, X. M. Zhang, S. Zhang, L. S. Ding and B. J. Li, Polym. Chem., 2015, 6, 2098-2107.

39 J. Ren, Y. X. Zhang, J. Zhang, H. J. Gao, G. Liu, R. J. Ma, Y. L. An, D. L. Kong and L. Q. Shi, Biomacromolecules, 2013, 14, 3434-3443.

40 M. S. Aw, M. Kurian and D. Losic, Chem.-Eur. J., 2013, 19, 12586-12601. 